\title{
Evaluation of success criteria for temporomandibular joint arthrocentesis
}

\author{
Onur Yilmaz ${ }^{1}$, Celal Candirli', Emre Balaban ${ }^{1}$, Mehmet Demirkol ${ }^{2}$ \\ ${ }^{\prime}$ Department of Oral and Maxillofacial Surgery, Faculty of Dentistry, Karadeniz Technical University, Trabzon, \\ ${ }^{2}$ Department of Oral and Maxillofacial Surgery, Faculty of Dentistry, Gaziantep University, Gaziantep, Turkey
}

\begin{abstract}
J Korean Assoc Oral Maxillofac Surg 2019;45:15-20)
Objectives: The aim of this study was to use four sets of success criteria to evaluate the outcomes of arthrocentesis treatment with hyaluronic acid injection in patients with internal derangement (ID) of the temporomandibular joint (TMJ).

Materials and Methods: The study included 40 patients diagnosed with unilateral Wilkes stage III TMJ dysfunction. Clinical parameters, including maximum mouth opening (MMO) and pain during function, were evaluated preoperatively, 6 months, and 1 year after TMJ arthrocentesis. Outcomes were assessed and compared using four sets of success criteria from the following: the American Association of Oral and Maxillofacial Surgeons (AAOMS; MMO $\geq 35 \mathrm{~mm}$ and visual analogue scale [VAS] score $\leq 3$ ), Murakami et al.'s criteria (MMO $>38 \mathrm{~mm}$ and VAS score $<2$ ), Emshoff and Rudisch criteria ( $\mathrm{MMO} \geq 35 \mathrm{~mm}$ and $>50 \%$ pain reduction), and patient self-reports (self-evaluation of treatment as successful or unsuccessful).

Results: Significant improvements in MMO and pain reduction during function were observed between the preoperative period and 6 months and 1 year postoperatively $(P<0.01)$. The success rates of treatment determined using AAOMS (52.5\%), Emshoff and Rudisch criteria (57.5\%), and selfreported patient criteria (40.0\%) were similar. Application of the Murakami et al. criteria reported the lowest success rate $(12.5 \%)$.

Conclusion: The AAOMS and Emshoff and Rudisch criteria are consistent with patient expectations and can be used to assess treatment efficacy.
\end{abstract}

Key words: Temporomandibular joint, Arthrocentesis, Temporomandibular joint disorders, Hyaluronic acid

[paper submitted 2018. 1. 19 / revised 2018. 4. 18 / accepted 2018. 5. 6]

\section{Introduction}

Temporomandibular joint (TMJ) dysfunction is caused by a wide variety of diseases, ranging from disc-dependent to degenerative conditions. All these diseases include functional difficulties and pain in the joint ${ }^{1,2}$. They cause various joint symptoms, most frequently limitations in and pain during mouth opening, and may also lead to degenerative changes in the cartilage and bone structures of the joint ${ }^{1-3}$. The most frequent cause of TMJ dysfunction is internal derangement (ID), which refers to alteration of the joint's normal path of

\section{Onur Yilmaz}

Department of Oral and Maxillofacial Surgery, Faculty of Dentistry, Karadeniz Technical University, 61080 Trabzon, Turkey

TEL: +90-462-3774811 FAX: +90-462-3253017

E-mail: onuryilmaz590@hotmail.com

ORCID: https://orcid.org/0000-0002-0460-7649

(c) This is an open-access article distributed under the terms of the Creative Commons Attribution Non-Commercial License (http://creativecommons.org/ licenses/by-nc/4.0/), which permits unrestricted non-commercial use, distribution, and reproduction in any medium, provided the original work is properly cited.

Copyright (C) 2019 The Korean Association of Oral and Maxillofacial Surgeons. All rights reserved. motion, largely involving the function of the articular dise ${ }^{1,3,4}$. The severity of ID is assessed using the Wilkes classification, which consists of five stages ranging from symptom-free slight forward displacement to degenerative arthritic changes accompanied by severe symptoms ${ }^{5}$.

The most frequently used initial treatments for ID of the TMJ are medication, occlusal splinting, and arthrocentesis ${ }^{6-8}$. Attempts to use these conservative methods prior to performing open joint surgery has become the standard clinical practice ${ }^{3,9}$. The area of indication for arthrocentesis is especially broad $^{6-9}$. Many studies have shown that arthrocentesis is an effective treatment for disc displacement without reduction $^{4,10}$. Arthrocentesis is extremely effective in eliminating joint adhesions, removing inflammatory cytokines, correcting joint function in the early stage of disease, and reducing pain $^{7,8,11}$. However, whether its effects persist over the long term is controversial; indeed, in most cases, it cannot address disc displacement adequately, and it does not contribute to the regeneration of degenerated joint structures ${ }^{2,12-14}$. Surprisingly, several articles refer to the importance of arthrocentesis, even for catastrophically degenerated joints. 
Many studies have used a decrease in pain values and an increase in mouth opening as success criteria without considering patient opinions. For example, a significant reduction in the visual analogue scale (VAS) score for pain from a preoperative value of 7 to a postoperative value of 5 reflects treatment success. However, a VAS pain score of 5 is indicative of disorder, and patients experiencing such pain probably do not consider treatment to have been successful; for this reason, a decrease in pain to a certain level does not mean that the treatment was completely successful.

Despite the publication of several sets of success criteria and their application in many studies, no clear standard has been established. The purpose of this study was to use different success criteria to evaluate the success of arthrocentesis with hyaluronic acid injection in patients with Wilkes stage III TMJ dysfunction.

\section{Materials and Methods}

This retrospective study included 40 patients with TMJ internal disorders who were diagnosed with unilateral localized TMJ pain and restricted mouth opening (disc displacement without reduction). All joints were evaluated clinically and radiologically (panoramic radiography and magnetic resonance imaging), and the Wilkes classification was used to determine the severity of TMJ disease. For standardization, only patients with Wilkes stage III dysfunction were included in the study. Wilkes stage 3 includes joint tenderness, limited mouth opening, frequent pain, non-reducing disc displacement, deformity/prolapse of disc (moderate to marked thickening of posterior edge), and no bone changes. Panoramic radiography confirmed that there was no change in hard tissue and the disk position was assessed by magnetic resonance imaging. Only patients with complete records from the treatment and follow-up periods through the beginning of the study were included. The inclusion criteria were patients with Wilkes stage III TMJ disorders, pain in the TMJ during mandibular movement and function, and unassisted maximum mouth opening (MMO) $<35 \mathrm{~mm}$. The exclusion criteria were degenerative joint disease, collagen vascular disease, diagnosis of TMJ myalgia, previous open TMJ surgery, pregnancy, history of acute trauma, and medical contraindications.

All patients were treated at the Department of Oral and Maxillofacial Surgery, Faculty of Dentistry, Karadeniz Technical University (Trabzon, Turkey) between 2016 and 2017. This study was conducted in compliance with the principles of the Declaration of Helsinki and with the approval of the
Human Ethics Committee of Karadeniz Technical University (2015/78). Patients provided written consent after the study procedures had been explained to them in detail.

\section{Clinical assessment}

The same clinician assessed clinical parameters, including pain during function and $\mathrm{MMO}$, preoperatively (T0), 6 months after treatment (T1), and 12 months after treatment (T2). Pain during function was evaluated using VAS ranging from 0 (no pain) to 10 (the most severe pain ever experienced) and reflects the maximum pain occurring during all mandibular movements. MMO was measured as the distance between the incisal edges of the upper and lower central incisors. The success of arthrocentesis with hyaluronic acid injection as the treatment for ID of the TMJ was evaluated using the following four sets of criteria:

- The criteria proposed by the American Association of Oral and Maxillofacial Surgeons (AAOMS), in which treatment is considered to be successful in the presence of mild or no pain (VAS score $\leq 3$ ) and an MMO $\geq 35 \mathrm{~mm}$ at 12 months after treatment ${ }^{15}$

- The criteria suggested by Murakami et al. ${ }^{13}$, comprising an $\mathrm{MMO}>38 \mathrm{~mm}$ and a VAS pain score $<2$;

- The criteria suggested by Emshoff and Rudisch ${ }^{16}$, comprising an $\mathrm{MMO} \geq 35 \mathrm{~mm}$ and $\mathrm{a}>50 \%$ pain reduction; and

- Patient treatment self-evaluation as successful or unsuccessful.

\section{Treatment procedure}

The same surgeon performed all arthrocentesis procedures under local anesthesia; each procedure was completed in a single session. Local anesthetic (4\% articaine hydrochloride with 1:200,000 epinephrine) was injected into the superior joint space and overlying tissues by gently withdrawing the needle, followed by lysis and lavage of the upper joint compartment using the technique described by Nitzan et al. ${ }^{17}$. A 21-gauge needle was then inserted into the upper joint space, $10 \mathrm{~mm}$ anterior to the tragus and $2 \mathrm{~mm}$ inferior to an imaginary line connecting the tragus and lateral canthus. Two milliliters of saline were administered to release fibrous adhesions, distend the joint space, and facilitate insertion of a second 21-gauge needle into the distended compartment. The joint was irrigated with $250 \mathrm{~mL}$ of ringer lactate solution, establishing free flow of the irrigating solution. Finally, $1 \mathrm{~mL}$ of hyaluronic acid (Orthovisc; Biomeks, Istanbul, Turkey) was 
injected into the upper joint space. After treatment, patients were prescribed an analgesic, a muscle relaxant, and soft diet for 1 week, and patients were advised to completely rest the joint for 3 days. No other treatments were performed during the follow-up period.

\section{Statistical analysis}

Statistical analysis was performed with the SPSS software (ver. 17.0 for Windows; SPSS, Chicago, IL, USA). Data on age were reported as means with standard deviations, and VAS scores and MMO values were reported as medians and ranges. The sex distribution is reported as the ratio of males to females. The normality of VAS and MMO data was tested using the Kolmogorov-Smirnov test. Differences in VAS scores and MMO values between time points were analyzed using the Wilcoxon signed rank test. Spearman's correlation analysis was used to compare treatment outcomes according to the AAOMS ${ }^{15}$, Murakami et al. ${ }^{13}$, Emshoff and Rudisch ${ }^{16}$, and patient criteria at each time point. The confidence level was set to $95 \%$.

\section{Results}

Of the 40 patients included in the study, 32 were female and 8 were male. The mean age of the patients was 31.73 years (range, 18-57 years). All patients tolerated arthrocentesis well. Aside from temporary facial paralysis due to the administration of local anesthesia, no complications were observed.

Table 1. Comparison of preoperative and 12-month follow-up data $(n=40)$

\begin{tabular}{lcccl}
\hline & $\begin{array}{c}\text { Preoperative } \\
(\mathrm{T} 0)\end{array}$ & $\begin{array}{c}6 \text { months } \\
(\mathrm{T} 1)\end{array}$ & $\begin{array}{c}12 \text { months } \\
(\mathrm{T} 2)\end{array}$ & $P$-value \\
\hline MMO (mm) & $35(20-43)$ & $36(28-44)$ & $38(30-45)$ & $0.001^{1}$ \\
Pain during & $7(5-8)$ & $4(2-7)$ & $3(2-7)$ & $0.0001^{2}$ \\
function (VAS) & & & & \\
\hline
\end{tabular}

(MMO: maximum mouth opening, VAS: visual analogue scale)

${ }^{1}$ There were statistically significant differences between pretreatment and other periods $(P<0.01)$, but there were no statistically significant differences between 6 months and 12 months $(P>0.05)$.

${ }^{2}$ There were statistically significant differences between pretreatment and other periods $(P<0.001)$, but there were no statistically significant differences between 6 months and 12 months $(P>0.05)$.

Values are presented as median (range).

Onur Yilmaz et al: Evaluation of success criteria for temporomandibular joint arthrocentesis. J Korean Assoc Oral Maxillofac Surg 2019

\section{Effects of arthrocentesis with hyaluronic acid injection on pain scores and MMO}

The reduction in pain scores from preoperative to 6 months ( 7 vs $4 ; P<0.001$ ) and preoperative to 12 months ( 7 vs 3 ; $P<0.001$ ) were found to be statistically significant.(Table 1 ) The reduction in pain scores from 6 to 12 months was not statistically significant $(P>0.05)$. (Table 1$) \mathrm{MMO}$ values were significantly higher 12 months and 6 months after treatment than at baseline $(P<0.01)$.(Table 1) The difference between 12 months and 6 months was not statistically significant $(P>0.05)$. (Table 1) These results indicate that treatment was successful 6 months and 12 months postoperatively.

\section{Success of arthrocentesis with hyaluronic acid injection according to various criteria}

At 12 months after treatment, 16 patients reported that the treatment was successful, and 24 patients reported that it was unsuccessful. According to the AAOMS criteria ${ }^{15}$, the treatment was successful in 21 patients and unsuccessful in 19 patients. According to the Murakami et al. ${ }^{13}$ criteria, the treatment was successful in 5 patients and unsuccessful in 35 patients. According to the Emshoff and Rudisch ${ }^{16}$ criteria, treatment was successful in 23 patients and unsuccessful in 17 patients.(Table 2 )

Correlations were observed between all pairs of success ratings except between those obtained by patient self-evaluation and the Murakami et al. ${ }^{13}$ criteria. The strongest correlation was observed between the ratings obtained with the Emshoff and Rudisch ${ }^{16}$ and the AAOMS ${ }^{15}$ criteria $(r=0.904, P<0.01)$, followed by those obtained with patient self-evaluation and the AAOMS criteria $(r=0.572, P<0.01)$. (Table 3$)$ These results show that ratings obtained with the AAOMS and Emshoff and Rudisch criteria are consistent with patient ratings, with the AAOMS criteria aligning more closely than the Emshoff and Rudisch criteria with patient self-evaluations.

Although pain scores decreased significantly and MMO

Table 2. Distribution of patients according to different criteria, classified as successful/unsuccessful

\begin{tabular}{lcccc}
\hline & $\begin{array}{c}\text { Emshoff and } \\
\text { Rudisch }^{16}\end{array}$ & $\begin{array}{c}\text { Patient } \\
\text { self-report }\end{array}$ & AAOMS $^{15}$ & $\begin{array}{c}\text { Murakami } \\
\text { et al. }\end{array}$ \\
\hline Successful & $23(57.5)$ & $16(40.0)$ & $21(52.5)$ & $5(12.5)$ \\
Unsuccessful & $17(42.5)$ & $24(60.0)$ & $19(47.5)$ & $35(87.5)$ \\
Total & $40(100)$ & $40(100)$ & $40(100)$ & $40(100)$ \\
\hline
\end{tabular}

Values are presented as number (\%).

Onur Yilmaz et al: Evaluation of success criteria for temporomandibular joint arthrocentesis. J Korean Assoc Oral Maxillofac Surg 2019 
Table 3. Correlation of different success criteria

\begin{tabular}{|c|c|c|c|c|c|}
\hline & & $\begin{array}{l}\text { Murakami } \\
\text { et al. }^{13}\end{array}$ & $\mathrm{AAOMS}^{15}$ & $\begin{array}{l}\text { Emshoff } \\
\text { and } \\
\text { Rudisch }^{16}\end{array}$ & $\begin{array}{l}\text { Patient } \\
\text { self- } \\
\text { report }\end{array}$ \\
\hline \multirow{2}{*}{$\begin{array}{l}\text { Murakami } \\
\text { et al. }^{13}\end{array}$} & $r$ & 1.000 & 0.360 & 0.325 & 0.309 \\
\hline & $P$-value & - & $0.023^{*}$ & $0.041 *$ & 0.053 \\
\hline \multirow{2}{*}{$\mathrm{AAOMS}^{15}$} & $r$ & 0.360 & 1.000 & 0.904 & 0.572 \\
\hline & $P$-value & $0.023 *$ & - & $0.000 * *$ & $0.000 * *$ \\
\hline \multirow{2}{*}{$\begin{array}{l}\text { Emshoff and } \\
\text { Rudisch }^{16}\end{array}$} & $r$ & 0.325 & 0.904 & 1.000 & 0.496 \\
\hline & $P$-value & $0.041 *$ & $0.000 * *$ & - & $0.001 * *$ \\
\hline \multirow{2}{*}{$\begin{array}{l}\text { Patient self- } \\
\text { report }\end{array}$} & $r$ & 0.309 & 0.572 & 0.496 & 1.000 \\
\hline & $P$-value & 0.053 & $0.000 * *$ & $0.001 * *$ & . \\
\hline
\end{tabular}

*Correlation is significant at the 0.05 level.

**Correlation is significant at the 0.01 level.

Onur Yilmaz et al: Evaluation of success criteria for temporomandibular joint arthrocentesis. J Korean Assoc Oral Maxillofac Surg 2019

values increased significantly 1 year postoperatively, the treatment was rated as unsuccessful by a large number of patients. Application of the Murakami et al. ${ }^{13}$ criteria yielded an especially low treatment success rate.

\section{Discussion}

Since the introduction of arthrocentesis, the indication range for this procedure has broadened ${ }^{3,4,9,10}$. Since arthrocentesis is a minimally invasive surgical procedure, it is preferred for the treatment of ID of the $\mathrm{TMJ}^{10,11,18,19}$. When it is performed with pressure, the elimination of adhesions in the joint is the key element in the success of this method. Joint mobility increases as a result of the elimination of adhesions ${ }^{19-21}$. The elimination of inflammatory cytokines by arthrocentesis plays a role in pain reduction ${ }^{20,22}$. Many studies have confirmed the positive effects of arthrocentesis, especially in the improvement of pain and $\mathrm{MMO}^{20,21,23}$.

The indications for arthrocentesis vary among researchers. Monje-Gil et al. ${ }^{24}$ reported that arthrocentesis is more successful in acute locking situations than in cases of chronic disc displacement without reduction. Al-Baghdadi et al. ${ }^{25}$ reported that arthrocentesis could be more successful in terms of clinical parameters in cases with the anchored disc phenomenon and had claimed that the procedure's long-term success in patients with non-chronic reduction disc displacement could be due to remodeling. Several studies have documented the success of arthrocentesis for treating disc displacement with reduction accompanied by severe pain and sound ${ }^{23,26}$. The literature also supports the long-term efficacy of arthrocentesis in patients with ID. Alpaslan et al. ${ }^{27}$ had evaluated pain, dysfunction, and MMO after arthrocentesis in patients with ID of the TMJ. After an average follow-up period of 22 months (range, 3-60 months), they observed significantly reduced pain and dysfunction and significantly increased MMO. Dimitroulis et al. ${ }^{18}$ considered arthrocentesis to be an alternative to more invasive TMJ procedures. In a review, Monje-Gil et al. ${ }^{24}$ reported that arthrocentesis was successful in $80 \%$ of studies examined. These reports of the procedure's success have prompted surgeons to test the applicability of arthrocentesis for more severe joint diseases. Some authors have reported that arthrocentesis is successful, as measured by function and pain scores, even in patients with advanced degenerative disease ${ }^{8,28}$. However, arthrocentesis is performed more widely in patients with Wilkes stage III and IV dysfunction. For this reason, patients with Wilkes stage III dysfunction were included in this study and followed for 1 year.

Eighty percent of participants in our study were female. Joint disorders are observed more frequently in women than in men, and the data obtained in our study are similar to those reported in other studies ${ }^{3,29-32}$. At 1 year after arthrocentesis with hyaluronic acid injection, the mean VAS score had decreased significantly, and the mean MMO value had increased significantly. These results are in agreement with those of many other studies ${ }^{7,9,23}$. According to some authors, Wilkes stage III dysfunction is an indication for TMJ surgery. McCain et al. ${ }^{33}$ had reported that arthroscopic disc repositioning is an effective treatment modality in patients with Wilkes stage II and III disorders. Bronstein and Merrill ${ }^{34}$ had reported that, according to the Wilkes classification, the success rate of arthroscopic surgery was higher in patients with less advanced dysfunction. However, Nan et al. ${ }^{7}$ had reported that arthrocentesis effectively reduced pain and articular effusion in the short term in Wilkes stage III cases. Ungor et al. ${ }^{35}$ had reported that arthrocentesis was a reliable treatment modality in patients with Wilkes stage II and III dysfunction. Similarly, our results show that arthrocentesis with hyaluronic acid injection was successful at 1 year after surgery in patients with Wilkes stage III dysfunction.

According to many reviews, the success of arthrocentesis has been determined according to increased MMO and decreased pain scores ${ }^{20,23,24,31}$. However, the reduction of pain to a certain level does not indicate that treatment had been completely successful and that no further treatment is required. Patient ratings of treatment success provide a different perspective. To determine the appropriate success criteria, we evaluated arthrocentesis using four sets of criteria. In developing their criteria, Murakami et al. ${ }^{13}$ and Emshoff and Rudisch ${ }^{16}$ had made evaluation of arthrocentesis success more detailed and systematic. For this reason, these criteria were applied in this study. The AAOMS defines diagnostic 
and treatment criteria for maxillofacial surgery that many researchers use as a reference ${ }^{15,36}$. For this reason, the AAOMS criteria for the success of arthrocentesis were also evaluated in this study. Additionally, patient assessments of whether the treatment was successful were compared with the ratings obtained using the aforementioned criteria. To our knowledge, no other study in the literature has involved the evaluation of treatment success according to different criteria or has investigated whether these criteria are consistent with patient expectations. The results of this study reveal which criteria are better for evaluating treatment success.

At 1 year postoperatively, arthrocentesis was rated as unsuccessful in $60.0 \%, 47.5 \%, 87.5 \%$, and $42.5 \%$ of patients according to patient self-reports and the AAOMS, Murakami et al. ${ }^{13}$, and Emshoff and Rudisch ${ }^{16}$ criteria, respectively. Although arthrocentesis had positive effects on pain scores and MMO values, its success is debatable in light of these results.

Significant correlations were observed among ratings obtained with the AAOMS, Emshoff and Rudisch ${ }^{16}$, and Murakami et al. ${ }^{13}$ criteria. Ratings obtained with the AAOMS and Emshoff and Rudisch criteria were strongly correlated with patient self-reports, whereas those obtained with the Murakami et al. ${ }^{13}$ criteria were not correlated with patient self-evaluations. Thus, the use of the AAOMS and Emshoff and Rudisch ${ }^{16}$ criteria yielded results that were more consistent with patient treatment expectations. The Murakami et al. ${ }^{13}$ criteria appeared to include extremely high expectations, as ratings obtained with these criteria indicated that treatment was successful in only $12.5 \%$ of patients at 1 year after surgery. On the other hand, although ratings obtained with the AAOMS and Emshoff and Rudisch ${ }^{16}$ criteria were significantly correlated, the AAOMS criteria were more consistent with patient reactions to the treatment.

The literature contains other success criteria used to assess the efficacy of treatment of TMJ disorders. Guarda-Nardini et al. ${ }^{37}$ examined the effects of arthrocentesis with hyaluronic acid injection on degenerative joint disorders using success criteria that included a 50\% decline in the pain score and obtained more objective data. Similarly, Holmlund et al. ${ }^{38}$ used their own criteria to evaluate the success of discectomy. In recent years, the indication range for arthrocentesis treatment has widened ${ }^{8,9,12,32}$. Determination of its success will clarify its effects on different pathologies.

The limitations of this study included the evaluation of treatment as successful/unsuccessful according to patient reactions but in the absence of a more detailed exploration of patient expectations. Patient pain thresholds and psychologi- cal characteristics should be taken into consideration. Other limitations of the study were that patients with disorders at different Wilkes stages were not evaluated and the sample size was small.

\section{Conclusion}

The AAOMS and Emshoff and Rudisch ${ }^{16}$ criteria can be considered appropriate for the evaluation of arthrocentesis success, as the criteria generated results that are in agreement with patient expectations. Further research is needed to make these criteria more systematic by including various parameters.

\section{ORCID}

Onur Yilmaz, https://orcid.org/0000-0002-0460-7649

Celal Candirli, https://orcid.org/0000-0003-4926-646X

Emre Balaban, https://orcid.org/0000-0001-5399-2152

Mehmet Demirkol, https://orcid.org/0000-0003-1973-0364

\section{Authors' Contributions}

O.Y., C.C., M.D., and E.B. designed the study. C.C. and O.Y. performed the treatments. O.Y. and M.D. collected the data in this study. E.B. and M.D. interpreted and analyzed the data. O.Y. and M.D. drafted the article. O.Y., C.C., and M.D. critically revised and aproved the article. All authors read and approved the final version of the manuscript.

\section{Ethics Approval and Consent to Participate}

This study approved by the Human Ethics Committee of Karadeniz Technical University (2015/78). Informed consent was obtained from all individual participants included in this study.

\section{Conflict of Interest}

No potential conflict of interest relevant to this article was reported.

\section{References}

1. Dolwick MF, Riggs RR. Diagnosis and treatment of internal derangements of the temporomandibular joint. Dent Clin North Am 1983;27:561-72.

2. List T, Jensen RH. Temporomandibular disorders: old ideas and 
new concepts. Cephalalgia 2017;37:692-704.

3. Giraddi GB, Siddaraju A, Kumar B, Singh C. Internal derangement of temporomandibular joint: an evaluation of effect of corticosteroid injection compared with injection of sodium hyaluronate after arthrocentesis. J Maxillofac Oral Surg 2012;11:258-63.

4. Bhargava D, Jain M, Deshpande A, Singh A, Jaiswal J. Temporomandibular joint arthrocentesis for internal derangement with disc displacement without reduction. J Maxillofac Oral Surg 2015;14:454-9.

5. Wilkes $\mathrm{CH}$. Internal derangements of the temporomandibular joint. Pathological variations. Arch Otolaryngol Head Neck Surg 1989;115:469-77.

6. Patel P, Idrees F, Newaskar V, Agrawal D. Sodium hyaluronate: an effective adjunct in temporomandibular joint arthrocentesis. Oral Maxillofac Surg 2016;20:405-10.

7. Nan Z, Guoliang J, Zhongyin W. [Clinical symptoms and temporomandibular joint disc deformity study of Wilkes III stage patients treated with arthrocentesis]. West Chin J Stomatol 2015;33:585-8. Chinese.

8. Emes Y, Arpınar IŞ, Oncü B, Aybar B, Aktaş I, Al Badri N, et al. The next step in the treatment of persistent temporomandibular joint pain following arthrocentesis: a retrospective study of 18 cases. J Craniomaxillofac Surg 2014;42:e65-9.

9. Leibur E, Jagur O, Voog-Oras Ü. Temporomandibular joint arthrocentesis for the treatment of osteoarthritis. Stomatologija 2015;17:113-7.

10. Diraçoğlu D, Saral IB, Keklik B, Kurt H, Emekli U, Ozçakar L, et al. Arthrocentesis versus nonsurgical methods in the treatment of temporomandibular disc displacement without reduction. Oral Surg Oral Med Oral Pathol Oral Radiol Endod 2009;108:3-8.

11. Talaat W, Ghoneim MM, Elsholkamy M. Single-needle arthrocentesis (Shepard cannula) vs. double-needle arthrocentesis for treating disc displacement without reduction. Cranio 2016;34:296-302.

12. Nitzan DW, Samson B, Better H. Long-term outcome of arthrocentesis for sudden-onset, persistent, severe closed lock of the temporomandibular joint. J Oral Maxillofac Surg 1997;55:151-7; discussion 157-8.

13. Murakami K, Hosaka H, Moriya Y, Segami N, lizuka T. Short-term treatment outcome study for the management of temporomandibular joint closed lock. A comparison of arthrocentesis to nonsurgical therapy and arthroscopic lysis and lavage. Oral Surg Oral Med Oral Pathol Oral Radiol Endod 1995;80:253-7.

14. Sembronio S, Albiero AM, Toro C, Robiony M, Politi M. Is there a role for arthrocentesis in recapturing the displaced disc in patients with closed lock of the temporomandibular joint? Oral Surg Oral Med Oral Pathol Oral Radiol Endod 2008;105:274-80; discussion 281.

15. American Association of Oral and Maxillofacial Surgeons. 1984 criteria for TMJ meniscus surgery. Chicago: American Association of Oral and Maxillofacial Surgeons; 1984:1-40.

16. Emshoff R, Rudisch A. Temporomandibular joint internal derangement and osteoarthrosis: are effusion and bone marrow edema prognostic indicators for arthrocentesis and hydraulic distention? J Oral Maxillofac Surg 2007;65:66-73.

17. Nitzan DW, Dolwick MF, Martinez GA. Temporomandibular joint arthrocentesis: a simplified treatment for severe, limited mouth opening. J Oral Maxillofac Surg 1991;49:1163-7; discussion 116870.

18. Dimitroulis G, Dolwick MF, Martinez A. Temporomandibular joint arthrocentesis and lavage for the treatment of closed lock: a followup study. Br J Oral Maxillofac Surg 1995;33:23-6; discussion 26-7.

19. Lee HS, Baek HS, Song DS, Kim HC, Kim HG, Kim BJ, et al. Effect of simultaneous therapy of arthrocentesis and occlusal splints on temporomandibular disorders: anterior disc displacement without reduction. J Korean Assoc Oral Maxillofac Surg 2013;39:1420.

20. Sharma A, Rana AS, Jain G, Kalra P, Gupta D, Sharma S. Evalu- ation of efficacy of arthrocentesis (with normal saline) with or without sodium hyaluronate in treatment of internal derangement of TMJ: a prospective randomized study in 20 patients. J Oral Biol Craniofac Res 2013;3:112-9.

21. Yura S, Totsuka Y, Yoshikawa T, Inoue N. Can arthrocentesis release intracapsular adhesions? Arthroscopic findings before and after irrigation under sufficient hydraulic pressure. J Oral Maxillofac Surg 2003;61:1253-6.

22. Kaneyama K, Segami N, Nishimura M, Sato J, Fujimura K, Yoshimura $H$. The ideal lavage volume for removing bradykinin, interleukin-6, and protein from the temporomandibular joint by arthrocentesis. J Oral Maxillofac Surg 2004;62:657-61.

23. Alpaslan GH, Alpaslan C. Efficacy of temporomandibular joint arthrocentesis with and without injection of sodium hyaluronate in treatment of internal derangements. J Oral Maxillofac Surg 2001;59:613-8; discussion 618-9.

24. Monje-Gil F, Nitzan D, González-Garcia R. Temporomandibular joint arthrocentesis. Review of the literature. Med Oral Patol Oral Cir Bucal 2012;17:e575-81.

25. Al-Baghdadi M, Durham J, Araujo-Soares V, Robalino S, Errington L, Steele J. TMJ disc displacement without reduction management: a systematic review. J Dent Res 2014;93:37S-51S.

26. Tuncel U. Repeated sodium hyaluronate injections following multiple arthrocenteses in the treatment of early stage reducing disc displacement of the temporomandibular joint: a preliminary report. J Craniomaxillofac Surg 2012;40:685-9.

27. Alpaslan C, Dolwick MF, Heft MW. Five-year retrospective evaluation of temporomandibular joint arthrocentesis. Int J Oral Maxillofac Surg 2003;32:263-7.

28. Nitzan DW, Svidovsky J, Zini A, Zadik Y. Effect of arthrocentesis on symptomatic osteoarthritis of the temporomandibular joint and analysis of the effect of preoperative clinical and radiologic features. J Oral Maxillofac Surg 2017;75:260-7.

29. Warren MP, Fried JL. Temporomandibular disorders and hormones in women. Cells Tissues Organs 2001;169:187-92.

30. Ahmed N, Sidebottom A, O'Connor M, Kerr HL. Prospective outcome assessment of the therapeutic benefits of arthroscopy and arthrocentesis of the temporomandibular joint. Br J Oral Maxillofac Surg 2012;50:745-8.

31. Emshoff R. Clinical factors affecting the outcome of arthrocentesis and hydraulic distension of the temporomandibular joint. Oral Surg Oral Med Oral Pathol Oral Radiol Endod 2005;100:409-14.

32. Nitzan DW. Rationale and indications for arthrocentesis of the temporomandibular joint. Alpha Omegan 2003;96:57-63.

33. McCain JP, Hossameldin RH, Srouji S, Maher A. Arthroscopic discopexy is effective in managing temporomandibular joint internal derangement in patients with Wilkes stage II and III. J Oral Maxillofac Surg 2015;73:391-401.

34. Bronstein SL, Merrill RG. Clinical staging for TMJ internal derangement: application to arthroscopy. J Craniomandib Disord 1992;6:7-16.

35. Ungor C, Atasoy KT, Taskesen F, Pirpir C, Yilmaz O. Long-term outcome of arthrocentesis plus hyaluronic acid injection in patients with wilkes stage II and III temporomandibular joint internal derangement. J Craniofac Surg 2015;26:2104-8.

36. Al-Baghdadi M, Durham J, Steele J. Timing interventions in relation to temporomandibular joint closed lock duration: a systematic review of 'locking duration'. J Oral Rehabil 2014;41:24-58.

37. Guarda-Nardini L, Rossi A, Arboretti R, Bonnini S, Stellini E, Manfredini D. Single- or multiple-session viscosupplementation protocols for temporomandibular joint degenerative disorders: a randomized clinical trial. J Oral Rehabil 2015;42:521-8.

38. Holmlund AB, Axelsson S, Gynther GW. A comparison of discectomy and arthroscopic lysis and lavage for the treatment of chronic closed lock of the temporomandibular joint: a randomized outcome study. J Oral Maxillofac Surg 2001;59:972-7; discussion 977-8. 